\title{
Une nouvelle génération de cultures en industrie laitière : aspects microbiologiques, biotechnologiques et probiotiques des cultures de bactéries composées de souches sélectionnées d'origine intestinale humaine
}

\author{
JA Kurmann \\ Institut agricole de l'Etat de Fribourg, Grangeneuve, 1725 Posieux, Suisse
}

\begin{abstract}
Résumé - Le but de cette présentation est de donner une aide d'orientation sur certains aspects intéressant la recherche et le développement des propriétés probiotiques des cultures utilisées en industrie laitière. Les générations de cultures et de produits fermentés, passées, actuelles et futures sont caractérisées au plan microbiologique et des effets sanitaires. Pour donner un meilleur aperçu que celui présenté jusqu'à présent, la valeur physiologique-nutritive des produits laitiers fermentés est traitée selon l'origine des effets. Pour la sélection de souches probiotiques, les hommes sains sont devenus des sources inépuisables. En revanche, les sources d'isolation de souches techniques sont de plus en plus limitées. La séparation du marché en cultures techniques banales et cultures probiotiques valorisées est prévisible. Les cultures de yogourt laissent entrevoir, quant à leurs propriétés probiotiques, des possibilités encore inexploitées. II serait recommandable de commencer à optimaliser la sélection (y compris l'utilisation industrielle) du point de vue pouvoir probiotique renforcé des souches. Cela éviterait une banalisation du yogourt dans le futur. L'utilisation des cultures probiotiques s'est élargie comme simple additif microbien (eg le beurre), comme multiplicateur de ferments probiotiques sans fermentation (eg certains yogourts aux bifidobactéries avec temps d'incubation court) et comme multiplicateur et fermentateur avec des degrés d'intensité différents (eg certains produits bifidus).
\end{abstract}

probiotique / sélection de cultures probiotiques / cultures de yogourt / valeur physiologiquenutritive / lait fermenté / biotechnologie alimentaire

Summary - A new generation of starter cultures in the dairy industry : microbiological, biotechnological and probiotic aspects of bacterial cultures consisting of strains selected from the human intestine. The aim of this review is to provide an orientation on certain aspects which concern the research into and development of probiotic properties of cultures used in the dairy industry. The past, present and future of fermented milk products have been examined at a microbiological and sanitary level. In order to provide a clearer survey than those made up to now, the physiological-nutritive value of fermented milk products has been examined according to the origin of the effects. Healthy humans provide an inexhaustible source for the selection of probiotic strains. However, the sources for the selection of technical strains are becoming increasingly more limited. The separation of the market into ordinary technical cultures and probiotic starter cultures is a possibility. 
Yoghurt starter cultures possess as yet unexploited possibilities regarding their probiotic properties. It is recommended that selection be optimised (including industrial utilisation) regarding the reinforced probiotic strength of the strains. The use of probiotic starter cultures has extended to microbial additive (eg butter), to developing probiotic cultures without fermentation (eg bifidus yoghurt with a short incubation period) and to developing and fermenting at different degrees of intensity (eg certain bifidus products).

probiotics / probiotic starter culture selection / yogourt culture / nutritional-physiological value / fermented milk / food biotechnology

\section{INTRODUCTION}

Différents aspects et différentes perspectives probiotiques d'une nouvelle (troisième) génération de cultures sont traitées. II s'agit essentiellement de cultures composées de souches humaines de bactéries intestinales sélectionnées, comme par exemple de bifidobactéries et de lactobacilles utilisées, pour la production de produits laitiers fermentés.

Le but de cette présentation est de donner des points de repère pour la recherche et le développement, ainsi que pour l'utilisation des cultures probiotiques en industrie laitière. Le consommateur "2000" demandera beaucoup plus que jusqu'à présent des aliments favorisant sa performance et sa santé corporelle et, de ce fait, élaborés avec des souches de bactéries probiotiques (Kurmann, 1986b).

Comme les recherches entreprises jusqu'à présent se concentrent surtout sur les propriétés techniques des cultures, la sélection selon des critères sanitaires ne se trouve qu'à ses débuts. Elle fut soulevée à différentes reprises par Râsic et Kurmann (1978), et Kurmann (1989a, b) pour le yogourt; par Râsic et Kurmann (1983), Kurmann (1988) et Kurmann et Râsic (1991) pour les bifidobactéries; par Fondén (1989) pour Lb acidophilus et par Kurmann (1989b) d'une manière générale pour des cultures probiotiques.
La méthode utilisée pour l'étude des différents aspects des cultures probiotiques de la troisieme génération est celle à la fois d'une analyse et d'une synthèse dans le cadre d'un travail de revue, ainsi que l'application de connaissances multidisciplinaires.

Au plan «matériel», on peut mentionner l'expérience acquise dans le domaine des produits laitiers fermentés. La corédaction de 3 monographies scientifiques originales : Râsic et Kurmann $(1978,1983)$ et Kurmann et al (1992), répandues à travers les continents, facilite une vue globale et la recherche de synthèse.

\section{ASPECTS MICROBIOLOGIQUES}

Une rétrospective et perspective de la recherche et du développement des produits laitiers fermentés frais et de leurs cultures est donnée dans le tableau I qui mentionne aussi les causes et les caractéristiques de cette évolution. Nous distinguons 4 générations. Actuellement, comme dans le futur, des produits de différentes générations se trouvent ensemble sur le marché.

Le marché des cultures va se séparer, dans l'avenir, en cultures techniques banales (cultures de deuxième génération) et cultures probiotiques nouvelles (cultures de troisième génération). 
Tableau I. Les générations des cultures et produits laitiers fermentés vues au plan des propriétés probiotiques.

\begin{tabular}{|c|c|c|}
\hline $\begin{array}{l}\text { Générations de } \\
\text { produits laitiers } \\
\text { fermentés }\end{array}$ & $\begin{array}{l}\text { Caractéristiques } \\
\text { microbiologiques } \\
\text { des cultures }\end{array}$ & $\begin{array}{l}\text { Caractéristiques } \\
\text { sanitaires des produits }\end{array}$ \\
\hline $\begin{array}{l}1^{\text {re }} \text { génération } \\
\text { début après } 8000 \text { av JC }\end{array}$ & $\begin{array}{l}\text { Cultures empiriques } \\
\text { - microflore non définie } \\
\text { - activité non définie }\end{array}$ & $\begin{array}{l}\text { Propriétés sanitaires } \\
\text { empiriques }\end{array}$ \\
\hline $\begin{array}{l}2^{\circ} \text { génération } \\
\text { début } \approx 1910\end{array}$ & $\begin{array}{l}\text { Cultures techniques } \\
\text { - microflore définie } \\
\text { - activité définie } \\
\text { - application du génie génétique }\end{array}$ & $\begin{array}{l}\text { Théorie de Metchnikoff (1903) } \\
\text { - attribution d'effets sanitaires } \\
\text { - priorité aux propriétés } \\
\text { techniques fermentatives }\end{array}$ \\
\hline $\begin{array}{l}3^{\theta} \text { génération } \\
\text { début } \approx \\
\text { par Rettger (1921) }\end{array}$ & $\begin{array}{l}\text { Cultures probiotiques } \\
\text { - bactéries intestinales humaines } \\
\text { sélectionnées } \\
\text { - autres micro-organismes }\end{array}$ & $\begin{array}{l}\text { Probiotiques } \\
\text { - effets multiples } \\
\text { - investigations actives } \\
\text { - effets ponctuels }\end{array}$ \\
\hline $\begin{array}{l}4^{e} \text { génération } \\
\text { début après l'an } 2000\end{array}$ & $\begin{array}{l}\text { Cultures probiotiques } \\
\text { et thérapeutiques } \\
\text { - sélection de souches améliorées } \\
\text { - application du génie génétique }\end{array}$ & $\begin{array}{l}\text { Probiotiques } \\
\text { perfectionnées } \\
\text { - propriétés renforcées } \\
\text { - formulation chimique } \\
\text { des produits pour renforcer }\end{array}$ \\
\hline
\end{tabular}

Source : Kurmann (1989b); révisé en 1992.

Les cultures probiotiques seront plutôt utilisées comme cultures complémentaires aux cultures acidifiantes ou comme simples additifs. Les cultures acidifiantes à base de ferments lactiques devront être adaptées aux cultures probiotiques (pas trop acidifiantes et postacidifiantes, dépourvues d'antagonisme actif, etc).

Il existe le risque que le yogourt comme produit, ainsi que ses cultures, deviennent banalisés, si on ne développe pas des propriétés sanitaires (Kurmann, 1989b). II est urgent d'étudier l'isolation de nouvelles souches, si possible d'origine humaine, de procéder à des essais pour l'étude du pouvoir probiotique des souches isolées, d'étudier la compatibilité avec les bactéries intestinales sélectionnées, etc.

\section{ASPECTS BIOTECHNOLOGIQUES}

Non seulement la sélection des souches bactériennes d'après des propriétés probiotiques, mais aussi le processus de fabrication doivent être adaptés à la production d'un probiotique (produits laitiers de la troisième génération).

Lors de la fermentation, la température d'incubation est de $37-38^{\circ} \mathrm{C}$ pour le développement de souches humaines de bactéries intestinales sélectionnées. C'est la température de l'homme qui est importante pour l'activité in vivo. En revanche, le yogourt et les souches animales sont incubées vers $42-43^{\circ} \mathrm{C}$.

La technique de fabrication doit être orientée vers la sauvegarde des propriétés 
probiotiques $(\mathrm{pH}$, température de stockage, emballage optimalisé, etc). L'assurance de qualité de l'usine laitière ne doit pas seulement s'occuper, par des essais cliniques des propriétés probiotiques au moment de la fabrication, mais également s'intéresser à leur maintenance jusqu'au moment de la consommation.

\section{ASPECT PROBIOTIQUE}

Les laits fermentés représentent une précieuse entité naturelle (Kurmann, 1984) ou système biologique vivant. Celle-ci peut être mieux expliquée que ce n'était le cas jusqu'à présent, par la distinction de différents degrés de la valeur physiologique nutritive selon l'origine des effets (tableau II) à savoir : 1) effets primaires provenant du lait (matière première); 2) effets secondaires (chimiques), provenant du métabolisme de fermentation; 3 ) effets tertiaires (probiotiques) provenant de cellules vivantes en transit massif.

Les micro-organismes employés dans la production de laits fermentés ont un effet probiotique (insuffisamment étudié, uni- ou multifactoriel). II peut fortement varier d'effets mesurables, mais sans valeur pratique, jusqu'aux effets de vraie importance prophylactiques-préthérapeutiques. Comme mentionné dans le sous-chapitre précédent, ces propriétés doivent se retrouver dans le produit fabriqué et au moment de la consommation (tâche des techniciens de la production et du service de l'assurance de qualité de l'usine).

Pour la sélection de souches d'une nouvelle génération de cultures composées de bactéries intestinales sélectionnées d'origine humaine on peut faire 2 observations principales.

D'abord, parmi la flore intestinale résidente (autochtone) de l'homme, Leclerc et Mossel (1989) distinguent 3 groupes de germes (la flore dominante, la flore sousdominante et la flore fluctuante). Les souches employées pour la production de laits fermentés devraient appartenir en premier lieu, à la flore dominante (eg Bifidobacterium spp, éventuellement aussi $L b$ acidophilus). En second lieu, on peut éventuellement prendre en considération des souches d'espèces appartenant à la flore sous-dominante (autres lactobacilles, certains streptocoques fécaux).

Ensuite, la flore des laits fermentés fait partie de la flore intestinale allochtone provenant de l'extérieur. Cette flore en transit massif, après l'ingestion, s'élimine progressivement au cours d'environ 5-10 j par

Tableau II. Les 3 degrés successifs et associés de valeur physiologique-nutritive des laits fermentés et l'origine des effets en aperçu.

Degré 1 : effets primaires (la matière première : le lait)

Composition chimique équilibrée, riche et comportant un large spectre de bioactivité. Excellent support et éventuellement renforcement des propriétés probiotiques.

Degré 2 : effets secondaires (Changements chimiques par le processus de croissance et de fermentation du lait et formation de métabolites bioactifs).

Prédigestion des macrocomposants variant selon la nature des cultures employées. Péristaltisme favorisé, teneur en glucose plus faible dans le sang, exoenzymes.

Degré 3 : effets tertiaires (Effets du transif massif des cellules bactériennes contenues dans les laits fermentés et transport d'enzymes associées aux cellule)

Effets prophylactiques-préthérapeutiques (probiotiques) complémentaires, avec une intensité différente selon les groupes variés de produits (voir tableau III, sous-groupes A, B, C).

Source : Kurmann (1986a); révisè 1992. 
exemple, sauf s'il y a colonisation (exemple démontré avec B longum 536 par Ballongue et al, 1992).

Dans le tableau III, nous donnons 3 exemples de groupes importants de laits fermentés avec survie différente après l'ingestion (laits fermentés du type cultured buttermilk, yoghourt et lait fermenté aux bifidobactéries) avec des effets probiotiques possibles qui sont croissants de groupe en groupe.

On peut relever que, pour le transit massif, les bactéries intestinales sélectionnées provenant de la flore dominante sont

Tableau III. Effets du transit massif des cellules bactériennes vivantes contenues dans les laits fermentés : degré 3 (tableau II), sous-groupes.

\section{Sous-groupes de laits fermentés et type de culture utilisé}

Survie lors du transit massif (excrétion fécale)
Effets probiotiques possibles, appréciations sommaires
31

Laits fermentés avec ferments lactiques mésophiles (types str lactiques mésophiles, cultured buttermilk)

32

Laits fermentés avec des ferments lactiques thermophiles (type culture de yogourt)
Presque nulle ou en nombre très réduit

En nomore réduit, à la limite d'action probiotique, qui peut passer en-dessous selon les souches utilisées

\author{
Ep : isolés, faibles \\ Am : non mesurable \\ Te : réduite \\ Co: nulle \\ $\mathrm{Ri}$ : positive variable \\ (Forsén, 1989) \\ $\mathrm{Fi}$ : inconnus (peu probables) \\ $\mathrm{Rg}$ : Kurmann (1989a,b)
}

Ep : positive, limités

Am : mesurable, limité

Te : eg ß-galactosidase

Co: nulle

$\mathrm{Ri}$ : positive

$\mathrm{Fi}$ : positifs, limités

Rg : Râsic et Kurmann (1978);

Syndifrais (1989)
Ep : multipliés
Am : mesurable
$\mathrm{Te}$ : effets mesurables
Co: dépend des souches :
331 nulle, 332 positive
(Ballongue et al, 1992)
$\mathrm{Ri}$ : positive, dépend des souches
$\mathrm{Fi}$ : positifs, dépend des souches
$\mathrm{Rg}$ : Kurmann et Râsic (1991)


des meilleurs candidats à des effets probiotiques et à des effets probiotiques multiples que les autres bactéries (meilleure survie du transit, activité sur la flore intestinale stressée, transport d'enzymes associées aux cellules, etc).

Pour l'interprétation des résultats, ce serait une erreur grave de vouloir extrapoler à toutes les espèces de souches d'un genre de bactéries, les propriétés qui ont été trouvées pour une souche particulière (voir Kurmann, 1989b, pour la sélection des souches et Ballongue et al, 1992, pour des essais cliniques avec des souches probiotiques).

\section{DISCUSSION}

La sélection des souches, la composition des cultures et la fabrication de produits fermentés deviennent de plus en plus une profession de santé avec des conséquences correspondantes pour la conduite d'une bonne pratique de préparation.

L'homme sain devient un réservoir inépuisable pour l'isolation de souches probiotiques. En revanche, les sources d'isolation de souches techniques sont en diminution, du fait que les produits à fermentation spontanée disparaissent de plus en plus dans le monde.

Un concept de sélection de souches de cultures selon des critères probiotiques est à développer et à discuter. II embrasse des propriétés d'une identification sûre, un enzymogramme des propriétés d'importance sanitaire in vivo et une étude clinique des propriétés in vitro. Aussi, la sélection des cultures de yogourt doit entrer dans sa phase dynamique de recherche et de développement et montrera encore des possibilités inexploitées.

Sur la base de la distinction de l'origine des effets physiologiques nutritifs, il faudra, dorénavant, mieux séparer les effets physiologiques observés selon leur origine, c'est-à-dire bien distinguer les effets provenant du lait, de la fermentation et ceux provenant des bactéries vivantes en transit massif. Cela n'était pas fait, dans la plupart des cas, jusqu'à présent.

L'utilisation des cultures probiotiques s'élargit des produits avec fermentation (certains laits fermentés assez longtemps incubés) aux produits avec multiplication limitée, mais sans fermentation (certains yogourts au bifidus avec temps d'incubation court), aux produits sans fermentation et développement conséquent, mais avec maintenance de l'activité des bactéries probiotiques (beurre, glaces, pâtes molles, laits fermentés mésophiles, laits de consommation doux, etc). II est aussi possible d'utiliser des souches probiotiques précieuses qui ne se cultivent pas ou trop difficilement sur du lait, mais qui survivent en nombre suffisant, dans les produits fabriqués.

A la lumière des connaissances et tendances nouvelles, une simple extrapolation du terme probiotique en alimentation animale à l'alimentation humaine suscite certaines confusions et imprécisions. De ce fait, nous définissons le terme probiotique spécialement en vue d'un supplément d'aliment humain (voir ci-dessous).

Un probiotique dans l'alimentation humaine est un produit laitier contenant des souches de micro-organismes (bactéries et/ou levures et/ou moisissures) définies et GRAS (generally recognized as safe). Les bactéries intestinales humaines sélectionnées sont de bons candidats pour un nombre plus large d'effets probiotiques (tableau III, groupe 3 ). Ces probiotiques sont préparés avec une technologie spéciale pour assurer la bioactivité et la maintenance de ceux-ci jusqu'à la date limite de stockage dans des conditions précises. Le transit massif de ces micro-organismes lors de l'ingestion peut provoquer des ef- 
fets sanitaires favorables d'ordres prophylactique et préthérapeutique. L'action probiotique positive de souches de microorganismes et produits probiotiques est testée par des essais cliniques sur l'homme.

\section{REFERENCES}

Ballongue J, Grill JP, Euloge P (1992) Action sur la flore de laits fermentés au Bifidobacterium. In: Actualité Bifidus II (Kurmann JA, ed). Institut agricole de l'Etat de Fribourg, Posieux, Suisse

Bouhnik Y (1990) Etude in vivo chez l'homme du devenir d'une souche de Bifidobacterium $s p$ ingerée dans un produit laitier fermenté. DEA Univ Paris-Sud, France

Fondén R (1989) Lactobacillus acidophilus. In : Les laits fermentés. Actualité de la recherche (Perrin-Boulvard M, ed). John Libbey Eurotext, Paris

Forsén R (1989) Characterization of antigenic surface structure of lactococci and their possible immunobiological effects. In : Fermented milks and health (NIZO ed). NIZO, Ede, Netherlands

Kurmann JA (1984) La production des laits fermentés dans le monde. In : Les laits fermentés. Document 179/18. Fédération internationale de laiterie, Bruxelles

Kurmann JA (1986a) Yogourt made from ewe's and goat's milk. In : Production and utilization of ewe's and goat's milk. Bull Féd Int Lait (Bruxelles) 202, 152-165

Kurmann JA (1986b) The future of market milk. N Eur Dairy J 9-10, 304-310

Kurmann JA (1988) Starter with selected intestinal bacteria. In: Fermented milks. science and technology. Bull Int Dairy Fed (Bruxelles) 227, 41-45

Kurmann JA (1989a) A new generation of fermented milk products with selected intestinal bacteria for a better nutritional-physiologigal effect. In : Neue Entwicklung bei den Sauermilchprodukten. Commission Suisse du lait, Liebefeld-Bern

Kurmann JA (1989b) Microorganismes des laits fermentés. Aspects généraux, les bactéries, les levures, les moisissures, les souches et les microorganismes moins connus. In : Les Laits Fermentés. Actualité de la recherche (M Perrin-Boulvard, ed). John Libbey Eurotext, London-Paris

Kurmann JA, Râsic JLj (1991) The healthy potential of products containing bifidobacteria. In: Therapeutic Properties of Fermented Milks (Robinson RK, ed). Elsevier Science Publisher, London

Kurmann JA, Râsic JLj, Kroger M (1992) Encyclopedia of Fermented Fresh Milk and Related Products. Van Nostrand Reinhold, New York

Leclerc H, Mossel, DAA (1989) Microbiologie, le tube digestif, l'eau et les aliments. Doin, Paris

Metchnikoff E (1903) Etudes sur la nature humaine. Essai de philosophie optimiste. Masson, Paris

Râsic JLj, Kurmann JA (1978) Yogourt. Scientific grounds, technology, manufacture and preparations. Technical food and dairy publishing house, Helsingborg, Sweden

Râsic JLj, Kurmann JA (1983) Bifidobacteria and their role. Birkhäuser-Verlag, Basel, Switzerland

Rettger LF, Cheplin HA (1921) The transformation of intestinal flora with special reference to the implantation of Bacillus acidophilus. Yale University Press, New Haven 